\title{
Correction: Whole-exome sequencing identifies a novel CCDC151 mutation, c.325GT (p.E109X), in a patient with primary ciliary dyskinesia and situs inversus
}

Weizhi Zhang ${ }^{1,2} \cdot$ Dongping $\mathrm{Li}^{1,2} \cdot$ Shijie Wei ${ }^{1,2} \cdot$ Ting Guo $^{1} \cdot$ Jian Wang ${ }^{1} \cdot$ Hong Luo $^{1} \cdot$ Yifeng Yang $^{1,2} \cdot$ Zhiping Tan $^{1,2}$

Published online: 10 June 2019

(C) The Author(s), under exclusive licence to The Japan Society of Human Genetics 2019

\section{Correction to: Journal of Human Genetics} https://doi.org/10.1038/s10038-018-0540-x published online 30 November 2018

In the originally version of this article, the author affiliation 1 was incomplete. Clinical Center for Gene Diagnosis and Therapy isn't an independent institution and belongs to The Second Xiangya Hospital of Central South University. The full name of affiliation 1 is Clinical Center for Gene Diagnosis and Therapy, The Second Xiangya Hospital of Central South University, Changsha, China.

The authors would like to apologize for any inconvenience caused.

2 Department of Cardiovascular Surgery, The Second Xiangya Hospital of Central South University, Changsha, China 